\title{
Controllability of co-operative Neumann parabolic systems
}

\author{
Mohammed Shehata \\ Department of Mathematics, Faculty of Science, Jazan University, Jazan, Kingdom of Saudi Arabia
}

Email address:

mashehata_math@yahoo.com

\section{To cite this article:}

Mohammed Shehata. Controllability of Co-Operative Neumann Parabolic Systems. Pure and Applied Mathematics Journal. Vol. 4, No. 1, 2015, pp. 32-38. doi: 10.11648/j.pamj.20150401.15

\begin{abstract}
In this communication, we introduce and study the various controllability problems for Neumann co-operative parabolic linear system involving Laplace operator with distributed or boundary controls and with observations belong to different spaces.
\end{abstract}

Keywords: Optimal Control Problem, Controllability, Solutions of Parabolic System, Co-Operative System

\section{Introduction}

Controllability is a mathematical problem, which consists in determining the targets to which one can drive the state of some dynamical system, by means of a control parameter present in the equation. Many physical systems such as quantum systems, fluid mechanical systems, wave propagation, diffusion phenomena, etc. are represented by an infinite number of degrees of freedom, and their evolution follows some partial differential equation. Finding active controls in order to properly influence the dynamics of these systems generate highly involved problems. The control theory for PDEs, and among this theory, controllability problems, is a mathematical description of such situations. Any dynamical system represented by a PDE, and on which an external influence can be described, can be the object of a study from this point of view. In 1978, D.L. Russell [1] made a rather complete survey of the most relevant results that were available in the literature at that time. In that paper, the author described a number of different tools that were developed to address controllability problems, often inspired and related to other subjects concerning partial

$$
\pi(t ; y, y)+c_{0}\|y\|_{H}^{2} \geq c_{1}\|y\|_{V}^{2}, \quad c_{0} \geq 0, c_{1}>0, \quad \text { for } y \in V, t \in[0, T] .
$$

Then, from [20] and [21], for given $f, y_{0}$ and $B$ be a bounded linear operator the following abstract systems:

$$
\begin{aligned}
& \left.\frac{d}{d t} y(t)+A(t) y(t)=f+B u, t \in\right] 0, T[ \\
& y(0)=y_{0}
\end{aligned}
$$

have a unique solution, we denote it by $y(t ; u) \in Y$. differential equations: multipliers, moment problems, nonharmonic Fourier series, etc.

Various types of controllability of linear abstract dynamical systems defined in a Banach or Hilbert spaces have been recently extensively explored by several authors (see e.g.[2]-[18]). More recently, J.-L. Lions introduced the so called Hilbert Uniqueness Method (H.U.M.; see [19]).

In this work, we will focus our attention on some special aspects of controllability problems for parabolic system involving Laplace operator. In order to explain the results we have in mind, it is convenient to consider the abstract form:

Let $V$ and $H$ be two real Hilbert spaces such that $V$ is a dense subspace of $H$. Identifying the dual of $H$ with $H$, we may consider $V \subset H \subset V^{\prime}$, where the embedding is dense in the following space. Let $A(t)$ ( $t \in] 0, T[$ ) be a family of continuous operators associated with a bilinear forms $\pi(t ; .,$.$) defined on V \times V$ which are satisfied Gårding's inequality

We also given an observation equation $z(u)=C y(u), \quad C \in L(Y: \mathrm{H}), \quad \mathrm{H}$ being a Hilbert space.

Definition 1. The system whose state is defined by (2)is said to be controllable if the observation $z(u)$ generates a dense (affine) subspace of the space of observations $H$.

In the above setting, the equation (2) is typically a partial differential equation, where the influence of $u$ can take multiple different forms: typically, $u$ can be an additional 
(force) term in the right-hand side of the equation, localized in a part of the domain; it can also appear in the boundary conditions; but other situations can clearly be envisaged (we will describe some of them).

A typical application of a parabolic equation is the heat;

$$
\left.\begin{array}{ll}
\frac{\partial y}{\partial t}=\Delta y+u & \text { in } Q=\Omega \times] 0, T[, \\
y(x, 0)=y_{0}(x) & \text { in } \Omega, \\
\frac{\partial}{\partial v} y(x, t)=0 & \text { on } \Sigma=\Gamma \times] 0, T[,
\end{array}\right\}
$$

where $\Omega \subset R^{N}$ is a bounded open domain with smooth boundary $\Gamma, \frac{\partial}{\partial v}$ is the normal derivative at $\Gamma$ and $y_{0}(x)$ is a given function in $L^{2}(\Omega)$.

The results in [20] partly overlap with results in [22] and they were shown that the system (3) (with $u \in L^{2}(Q)$ ) is controllable.

In our papers [23]-[27], the above results have been extended, the controllability questions related to the time optimal control problem of $n \times n$ co-operative parabolic or hyperbolic systems with distributed or boundary controls was considered.

In this paper, we will consider various time-optimal control problems for the following $n \times n$ Neumann co-operative linear parabolic system (here and everywhere below the vectors are denoted by bold letters and the index $i=1,2, \ldots, n)$ :

$$
\left.\begin{array}{lr}
\frac{\partial y_{i}}{\partial t}=(A(t) \mathrm{y})_{i}+u_{i}(x, t) & \text { in } Q, \\
y_{i}(x, 0)=y_{i, 0}(x) & \text { in } \Omega, \\
\frac{\partial}{\partial v} y_{i}(x, t)=v_{i}(x, t), & \text { on } \Sigma
\end{array}\right\}
$$

where $y_{i, 0}$, is a given functions, $u_{i}$ represents either a distributed control or a given function defined in $Q, v_{i}$ represents either a Neumann boundary control or a given function defined in $\Sigma$ and $A(t) \quad(t \in] 0, T[$ ) are a family of $n \times n$ continuous matrix operators,

$$
\begin{aligned}
& A(t) y=\left(\begin{array}{cccc}
\Delta+a_{1} & a_{12} & & a_{1 n} \\
a_{21} & \Delta+a_{2} & & a_{2 n} \\
\vdots & \vdots & \vdots & \vdots \\
a_{n 1} & a_{n 2} & & \Delta+a_{n}
\end{array}\right)\left(\begin{array}{c}
y_{1} \\
y_{2} \\
\vdots \\
y_{n}
\end{array}\right) \\
& \int_{Q} y_{i}\left[-\frac{\partial \varphi}{\partial t}+A^{*} \varphi_{i}\right] d x d t=\int_{\Omega} y_{i, 0} \varphi_{i}(x, 0) d x+\int_{Q} u_{i} \varphi_{i} d x d t+\int_{\Sigma} v_{i} \varphi_{i} d \Gamma d t
\end{aligned}
$$

\section{Definition 2.}

1. A real function $\mathrm{y}=\mathrm{y}(x, t)$ defined in $Q$ is said to be a weak solution for system (4) if

$$
\mathrm{y} \in C\left([0, T] ;\left(L^{2}(\Omega)\right)^{n}\right) \cap L^{2}\left(0, T ;\left(H^{1}(\Omega)\right)^{n}\right)
$$

with co-operative coefficient functions $a_{i}, a_{i j}$ satisfying the

$$
\left.\begin{array}{l}
a_{i}, a_{i j} \text { are positive functions in } L^{\infty}(Q), \\
a_{i j}(x, t) \leq \sqrt{a_{i}(x, t) a_{j}(x, t)} .
\end{array}\right\}
$$

A classical controllability problems consists in steering an initial vector state $\left(y_{1}(0), y_{2}(0), \ldots, y_{n}(0)\right)^{T}$ for system (4), with a distributed control $\mathrm{u}=\left(u_{1}, u_{2}, \ldots, u_{n}\right)^{T}$ belonging to $\left(L^{2}(Q)\right)^{n} \quad$ or with a Dirichlet boundary control $\mathrm{v}=\left(v_{1}, v_{2}, \ldots, v_{n}\right)^{T}$, belonging to $\left(L^{2}(\Sigma)\right)^{n}$ so that $R_{\mathrm{u}}(T)$ or $R_{\mathrm{v}}(T)$ generates a dense (affine) subspace of $H$. where

$$
\begin{aligned}
& R_{\mathrm{u}}(T)=\left\{\mathrm{y}(T ; \mathrm{u}): \mathrm{u} \in\left(L^{2}(Q)\right)^{n}\right\}, \\
& R_{\mathrm{v}}(T)=\left\{\mathrm{y}(T ; \mathrm{v}): \mathrm{v} \in\left(L^{2}(\Sigma)\right)^{n}\right\}
\end{aligned}
$$

\section{Solutions of Neumann Co-Operative}

This section is devoted to the analysis of the existence and uniqueness of solutions of system (4). We distinguish three lasses of solutions: strong, weak and ultra weak solutions . order $\ell$ which consists of all $\varphi \in L^{2}(\Omega)$ whose distributional derivatives $D^{q} \varphi \in L^{2}(\Omega), \quad|q| \leq \ell$, with the

$$
\begin{gathered}
<y, \varphi>_{H^{\ell}(\Omega)}=\sum_{|q| \leq \ell}<D^{q} y, D^{q} \varphi>_{L^{2}(\Omega)}, \\
q=\left\{q_{1}, \ldots, q_{N}\right\}, \quad|q|=q_{1}+\ldots+q_{N}, \\
D^{q}=D_{1}^{q_{1}} \ldots D_{N}^{q_{N}}, \quad D_{i}=\frac{\partial}{\partial x_{i}} .
\end{gathered}
$$

for all $\varphi \in C^{1}\left([0, T] ;\left(L^{2}(\Omega)\right)^{n}\right) \cap L^{2}\left(0, T ;\left(H^{1}(\Omega)\right)^{n}\right), \quad \varphi_{i}(x, T)=0, \quad \frac{\partial \varphi_{i}}{\partial \nu_{\Sigma}}=0$

2. We say that the function $y$ is a strong solution of (4) if 


$$
\mathrm{y} \in C\left([0, T] ;\left(H^{1}(\Omega)\right)^{n}\right) \cap L^{2}\left(0, T ;\left(H^{2}(\Omega)\right)^{n} \cap\left(H^{1}(\Omega)\right)^{n}\right) \cap H^{1}\left(0, T ;\left(L^{2}(\Omega)\right)^{n}\right)
$$

and the three conditions in (4) are satisfied almost everywhere in their corresponding domains.

3. We say that the function $y$ is an ultra solution of (4) or solution by transposition if

$$
\mathrm{y} \in C\left([0, T] ;\left(H^{-1}(\Omega)\right)^{n}\right) \cap L^{2}\left(0, T ;\left(L^{2}(\Omega)\right)^{n}\right)
$$

and

$$
\int_{Q} y_{i} f_{i} d x d t=<y_{i, 0}, \varphi_{i}(x, 0)>+\int_{\Sigma} v_{i} \varphi_{i} d \Gamma d t \quad \forall f_{i} \in L^{2}(Q)
$$

where $\varphi$ is the unique solution of the adjoint system

$$
\left.\begin{array}{l}
-\frac{\partial \varphi_{i}}{\partial t}-\left(A^{*}(t) \varphi\right)_{i}=f_{i} \quad \text { in } Q, \\
\varphi_{i}(x, T)=0 \quad \text { in } \Omega, \\
\frac{\partial \varphi_{i}}{\partial v}=0 . \text { on } \Sigma
\end{array}\right\}
$$

and $<$.. $>$ denotes the duality paring between $H^{-1}(\Omega)$ and $H^{1}(\Omega)$.

For $\quad \mathrm{y}=\left(y_{1}, y_{2}, \ldots, y_{n}\right)^{T}, \varphi=\left(\varphi_{1}, \varphi_{2}, \ldots, \varphi_{n}\right)^{T} \in\left(H^{1}(\Omega)\right)^{n}$ and $t \in] 0, T[$, let us define a family of continuous bilinear forms

$$
\begin{aligned}
\pi(t ; \mathrm{y}, \varphi) & =\sum_{i=1}^{n} \int_{\Omega}\left[\left(\nabla y_{i}\right)\left(\nabla \varphi_{i}\right)-a_{i}(x, t) y_{i} \varphi_{i}\right] d x-\sum_{i, j=1}^{n} \int_{\Omega} a_{i j}(x, t) y_{j} \varphi_{i} d x \\
& =\sum_{i=1}^{n} \int_{\Omega}\left[\left(-\Delta y_{i}\right)-a_{i}(x, t) y_{i}\right] \varphi_{i} d x-\sum_{i, j=1}^{n} \int_{\Omega} a_{i j}(x, t) y_{j} \varphi_{i} d x \\
& =\sum_{i=1}^{n}<-(A(t) \mathrm{y})_{i}, \varphi>_{L^{2}(\Omega)} .
\end{aligned}
$$

Lemma 1. If $\Omega$ is a regular bounded domain in $R^{N}$, with boundary $\Gamma$, and if $m$ is positive on $\Omega$ and smooth enough (in particular $m \in L^{\infty}(\Omega)$ ) then the eigenvalue problem:

$$
\left.\begin{array}{ll}
-\Delta y=\lambda m(x) y & \text { in } \Omega, \\
y=0 & \text { on } \Gamma
\end{array}\right\}
$$

possesses an infinite sequence of positive eigenvalues:

$$
\lambda_{1}(m) \int_{\Omega} m y^{2} d x \leq \int_{\Omega}|\nabla y|^{2} d x
$$

Proof. See[29].

Now, let

$$
\lambda_{1}\left(a_{i}\right) \geq n-1, \quad i=1,2, \ldots \ldots, n .
$$

Lemma 2. If (5) and (8) hold then, the bilinear form (6) satisfy the Gårding inequality

$$
\pi(t ; \mathrm{y}, \mathrm{y})+c_{0}\|\mathrm{y}\|_{\left(L^{2}(\Omega)\right)^{n}}^{2} \geq c_{1}\|\mathrm{y}\|_{\left(H^{1}(\Omega)\right)^{n}}^{2}, \quad c_{0}, c_{1}>0 .
$$

Moreover $\lambda_{1}(m)$ is simple, its associate eigenfunction $e_{m}$ is positive, and $\lambda_{1}(m)$ is characterized by:

Proof. In fact

$$
\begin{aligned}
\pi(t ; \mathrm{y}, \mathrm{y}) & =\sum_{i=1}^{n} \int_{\Omega}\left[\left|\nabla y_{i}\right|^{2}-a_{i}(x, t) y_{i}^{2}\right] d x-\sum_{i, j=1}^{n} \int_{\Omega} a_{i j}(x, t) y_{i} y_{j} d x \\
& \geq \sum_{i=1}^{n} \int_{\Omega}\left[\left|\nabla y_{i}\right|^{2}-a_{i}(x, t) y_{i}^{2}\right] d x-2 \sum_{i>j}^{n} \int_{\Omega} \sqrt{a_{i}(x, t) a_{j}(x, t)} y_{i} y_{j} d x .
\end{aligned}
$$

By Cauchy Schwarz inequality and (7),we obtain

$$
\begin{aligned}
\pi(t ; \mathrm{y}, \mathrm{y}) \geq & \sum_{i=1}^{n}\left(1-\frac{1}{\lambda_{1}\left(a_{i}\right)}\right) \int_{\Omega}\left|\nabla y_{i}\right|^{2} d x-\sum_{i=1}^{n} \int_{\Omega} a_{i} y_{i}^{2} d x \\
& -2 \sum_{i>j}^{n} \int_{\Omega}\left(\frac{1}{\sqrt{\lambda_{1}\left(a_{i}\right) \lambda_{1}\left(a_{j}\right)}}\right)\left(\int_{\Omega}\left|\nabla y_{i}\right|^{2} d x\right)^{\frac{1}{2}}\left(\int_{\Omega}\left|\nabla y_{j}\right|^{2} d x\right)^{\frac{1}{2}} \\
& \geq \sum_{i=1}^{n}\left(\frac{\lambda_{1}\left(a_{i}\right)-n+1}{\lambda_{1}\left(a_{i}\right)}\right) \int_{\Omega}\left|\nabla y_{i}\right|^{2} d x-\sum_{i=1}^{n} \int_{\Omega} a_{i} y_{i}^{2} d x
\end{aligned}
$$


Finally, from (8) we have (9).

Under the above lemma ( Lemma 2) and using the results of Lions [20] and Lions and Magenes [21] we can prove the following theorems:

Theorem 1. Assume that (5) and (8) hold. Then, problem (4)

1. has a unique weak solution if $u_{i} \in L^{2}\left(0, T ; H^{-1}(\Omega)\right), v_{i} \in H^{\frac{-1}{2}, \frac{-1}{4}}(\Sigma) \quad$ and $\quad y_{i, 0} \in L^{2}(\Omega)$.

Moreover, the mapping $t \rightarrow \mathrm{y}(t ; \mathrm{u})$ is continuous from $[0, T] \rightarrow\left(L^{2}(\Omega)\right)^{n}$.

2. has a unique strong solution if $u_{i} \in L^{2}\left(0, T ; L^{2}(\Omega)\right), v_{i} \in H^{\frac{1}{2}, \frac{1}{4}}(\Sigma) \quad$ and $\quad y_{i, 0} \in H^{1}(\Omega)$.

Moreover, the mapping $t \rightarrow \mathrm{y}(t ; \mathrm{u})$ is continuous from $[0, T] \rightarrow\left(H^{1}(\Omega)\right)^{n}$.

3. has a unique ultra solution if $u_{i} \in L^{2}\left(0, T ; L^{2}(\Omega)\right), v_{i} \in L^{2}\left(0, T ; L^{2}(\Gamma)\right) \quad$ and $\quad y_{i, 0} \in H^{-1}(\Omega)$. Moreover, the mapping $t \rightarrow \mathrm{y}(t ; \mathrm{u})$ is continuous from $[0, T] \rightarrow\left(H^{-1}(\Omega)\right)^{n}$.

where the sobolev space $H^{r, s}(Q), r, s \geq 0 \quad$ (see [21])is defined by

$$
H^{r, s}(Q)=H^{0}\left(0, T ; H^{r}(\Omega)\right) \bigcap H^{s}\left(0, T ; H^{0}(\Omega)\right)
$$

$H^{s}(0, T ; X)$ denotes the sobolev space of order $s$ of functions defined on $[0, T]$ and taking values in $X$.

Based on the above theorem, we may consider the following problems:

1. distributed control $\left.\left(\mathrm{u} \in\left(L^{2}(Q)\right)^{n}\right)\right)$ problem with observation in $C\left([0, T] ;\left(L^{2}(\Omega)\right)^{n}\right)$

2. distributed control $\left(\mathrm{u} \in\left(L^{2}(Q)\right)^{n}\right)$ ) problem with observation in $C\left([0, T] ;\left(H^{1}(\Omega)\right)^{n}\right)$

3. boundary control $\left(\mathrm{v} \in\left(L^{2}(\Sigma)\right)^{n}\right)$ ) problem with observation in $C\left([0, T] ;\left(L^{2}(\Omega)\right)^{n}\right)$

4. boundary control $\left(\mathrm{v} \in\left(L^{2}(\Sigma)\right)^{n}\right)$ ) problem with observation in $C\left([0, T] ;\left(H^{-1}(\Omega)\right)^{n}\right)$

\section{Controllability with Deferent Observations}

In this section, we take the deferent cases of observations:

\subsection{Distributed Control with Observation in $C\left([0, T] ;\left(L^{2}(\Omega)\right)^{n}\right)$}

Let $\mathrm{y}(t ; \mathrm{u})$ denote to the unique weak solution of (4), at time $t$ corresponding to a given control $\mathrm{u} \in\left(L^{2}(Q)\right)^{n}$ and a given functions $v_{i}, y_{i, 0}$, satisfying the hypothesis of Theorem 1, a). Occasionally, we write $\mathrm{y}(x, t ; \mathrm{u})$ when the explicit dependence on $x$ is required.

Let the observations be given by

$$
y_{i}(T ; u) \in L^{2}(\Omega)
$$

Theorem 2. Assume that (5) and (8) hold, then the system (4) with control $u \in\left(L^{2}(Q)\right)^{n}$ and observation (10) is controllable.

Proof. let us first remark that by translation we may always reduce the problem of controllability to the case were the system (4) with $y_{i, 0}=v_{i}=0$. We can show quit easily that (4) is controllable in $\left(L^{2}(\Omega)\right)^{n}$ if and only if the set of reachable states $R_{\mathrm{u}}(T)$ at any finite time $T>0$ is dense in $\left(L^{2}(\Omega)\right)^{n}$. By the Hahn-Banach theorem, this will be the case if

$$
\int_{\Omega} \psi_{i} y_{i}(T ; \mathrm{u}) d x=0, \quad \psi_{i} \in L^{2}(\Omega),
$$

for all $\mathrm{u} \in\left(L^{2}(Q)\right)^{n}$, implies that $\psi_{i}=0, i=1,2, \ldots, n$.

We introduce $\xi=\left(\xi_{1}, \xi_{2}, \ldots, \xi_{n}\right)^{T}$ as the solution of the following system

$$
\left.\begin{array}{ll}
-\frac{\partial \xi_{i}}{\partial t}-\left(A^{*}(t) \xi\right)_{i}=0 & \text { in } Q \\
\xi_{i}(T)=\psi_{i} \in L^{2}(\Omega) & \text { in } \Omega, \\
\frac{\partial}{\partial v} \xi_{i}=0 . & \text { on } \Sigma .
\end{array}\right\}
$$

The existence of a unique solution for system (12) can be proved using Theorem1,a), with an obvious change of variables.

Multiply the first equation in (12) by $y_{i}(t ; \mathrm{u})$ and using Green formula, we obtain the following identity:

$$
\begin{aligned}
0 & =\int_{Q}\left[-\frac{\partial \xi_{i}}{\partial t}-\left(A^{*}(t) \xi\right)_{i}\right] y_{i}(t ; \mathrm{u}) d x d t \\
& =-\left.\int_{\Omega} \xi_{i} y_{i}(t ; \mathrm{u})\right|_{0} ^{T} d x+\int_{Q} \xi_{i}\left[\frac{\partial}{\partial t} y_{i}(t ; \mathrm{u})+(A(t) y(t ; \mathrm{u}))_{i}\right] d x d t \\
& =-\int_{\Omega} \psi_{i} y_{i}(T ; \mathrm{u}) d x+\int_{Q} \xi_{i} u_{i} d x d t
\end{aligned}
$$

and so, if (11) holds, then

$$
\int_{Q} \xi_{i} u_{i} d x d t=0 \quad \forall u_{i} \in L^{2}(Q)
$$

hence $\xi_{i}=0$, and hence $\psi_{i}=0$.

\subsection{Distributed Control with Observation in $C\left([0, T] ;\left(H^{1}(\Omega)\right)^{n}\right)$}

In this section, let $\mathrm{y}(t ; \mathrm{u})$ denote to the unique strong solution of (4), at time $t$ corresponding to a given control $\mathrm{u} \in\left(L^{2}(Q)\right)^{n}$ and a given functions $v_{i} y_{i, 0}$, satisfying the 
hypothesis of Theorem 1,b). Let the observation be given by

$$
y_{i}(T ; u) \in H^{1}(\Omega)
$$

Theorem 3. Assume that (5) and (8) hold, then the system (4) with control $u \in\left(L^{2}(Q)\right)^{n} \quad$ and observation (13) is controllable.

Proof. We can reduce the problem of controllability to the case were the system (4) with $y_{i, 0}=v_{i}=0$. Here $\mathrm{y}(T ; \mathrm{u}) \in\left(H^{1}(\Omega)\right)^{n}$. To show the system is controllable let $\psi_{i} \in H^{-1}(\Omega)$ such that

$$
<\psi_{i}, y_{i}(T ; \mathrm{u})>=0 \quad \forall \mathrm{u} \in\left(L^{2}(Q)\right)^{n} .
$$

We introduce $\xi=\left(\xi_{1}, \xi_{2}, \ldots, \xi_{n}\right)^{T}$ as the solution of the following system

$$
\left.\begin{array}{ll}
-\frac{\partial \xi_{i}}{\partial t}-\left(A^{*}(t) \xi\right)_{i}=0 & \text { in } Q \\
\xi_{i}(T)=\psi_{i} \in H^{-1}(\Omega) & \text { in } \Omega, \\
\frac{\partial}{\partial v} \xi_{i}=0 . & \text { on } \Sigma .
\end{array}\right\}
$$

since $\psi_{i} \in H^{-1}(\Omega)$ ( it is sufficient to apply Theorem 1,c), after reversing sense of time) then there exist a unique ultra weak solution $\xi$ for (14) and

$$
\int_{Q} \xi_{i} u_{i} d x d t=<\psi_{i}, y_{i}(T ; \mathrm{u})>=0
$$

then $\xi_{i}=0$, and hence $\psi_{i}=0$.
Let $\mathrm{y}(t ; \mathrm{v})$ denote to the unique weak solution of (4), at time $t$ corresponding to a given control $\mathrm{v} \in\left(L^{2}(\Sigma)\right)^{n}$ and a given functions $u_{i}, y_{i, 0}$, satisfying the hypothesis of Theorem 1,a). Let the observation be given by

$$
y_{i}(T ; v) \in L^{2}(\Omega)
$$

Theorem 4. Assume that (5) and (8) hold, then the system (4) with control $v \in\left(L^{2}(\Sigma)\right)^{n}$ and observation (15) is controllable.

Proof. Here $\mathrm{y}(T ; \mathrm{v}) \in\left(L^{2}(\Omega)\right)^{n}$. To show the system is controllable let $\psi_{i} \in L^{2}(\Omega)$ such that

$$
\int_{\Omega} y_{i}(T ; \mathrm{v}) \psi_{i} d x=0 \quad \forall \mathrm{v} \in\left(L^{2}(\Sigma)\right)^{n} .
$$

We introduce $\xi=\left(\xi_{1}, \xi_{2}, \ldots, \xi_{n}\right)^{T}$ as the solution of the following system

$$
\left.\begin{array}{lc}
-\frac{\partial \xi_{i}}{\partial t}-\left(A^{*}(t) \xi\right)_{i}=0 & \text { in } Q \\
\xi_{i}(T)=\psi_{i} \in L^{2}(\Omega) & \text { in } \Omega, \\
\frac{\partial}{\partial v} \xi_{i}=0 . & \text { on } \Sigma .
\end{array}\right\}
$$

The existence of a unique solution for system (18) can be proved using Theorem1,a), with an obvious change of variables.

Multiply the first equation in (18) by $y_{i}(t ; \mathrm{v})$ and using Green's formula, we obtain the following identity:

\subsection{Boundary Control with Observation in}

$$
C\left([0, T] ;\left(L^{2}(\Omega)\right)^{n}\right)
$$

$$
\begin{aligned}
0 & =\int_{Q}\left[-\frac{\partial \xi_{i}}{\partial t}-\left(A^{*}(t) \xi\right)_{i}\right] y_{i}(t ; \mathrm{v}) d x d t \\
& =-\left.\int_{\Omega} \xi_{i} y_{i}(t ; \mathrm{v})\right|_{0} ^{T} d x+\int_{Q} \xi_{i}\left[\frac{\partial}{\partial t} y_{i}(t ; \mathrm{v})+(A(t) y(t ; \mathrm{v}))_{i}\right] d x d t+\int_{\Sigma} \xi_{i} \frac{\partial y_{i}}{\partial v} d \Sigma \\
& =-\int_{\Omega} y_{i}(T ; \mathrm{v}), \psi_{i} d x \int_{\Sigma} \frac{\partial \xi}{\partial v} y_{i} d \Sigma
\end{aligned}
$$

implies

Theorem $1, \mathrm{c})$. Let the observation be given by

$$
\int_{\Sigma} \xi v_{i} d \Sigma=0
$$

hence $\xi$ on $\Sigma$. The Cauchy data of $\xi$ on $\Sigma$ being zero, we conclude ( see [30]) $\xi=0$ and hence $\psi=0$.

\subsection{Boundary Control with Observation in $C\left([0, T] ;\left(H^{-1}(\Omega)\right)^{n}\right)$}

Let $\mathrm{y}(t ; \mathrm{v})$ denote to the unique ultra weak solution of (4), at time $t$ corresponding to a given control $\mathrm{v} \in\left(L^{2}(\Sigma)\right)^{n}$ and a given functions $u_{i}, y_{i, 0}$, satisfying the hypothesis of

Theorem 5. Assume that (5) and (8) hold, then the system (4) with control $v \in\left(L^{2}(\Sigma)\right)^{n}$ and observation (17) is controllable.

Proof. Here $\mathrm{y}(T ; \mathrm{v}) \in\left(H^{-1}(\Omega)\right)^{n}$. To show the system is controllable let $\psi_{i} \in H^{1}(\Omega)$ such that

$$
<y_{i}(T ; \mathrm{v}), \psi_{i}>=0 \quad \forall \mathrm{v} \in\left(L^{2}(\Sigma)\right)^{n} .
$$

We introduce $\xi=\left(\xi_{1}, \xi_{2}, \ldots, \xi_{n}\right)^{T}$ as the solution of the 
following system

$$
\left.\begin{array}{ll}
-\frac{\partial \xi_{i}}{\partial t}-\left(A^{*}(t) \xi\right)_{i}=0 & \text { in } Q, \\
\xi_{i}(T)=\psi_{i} \in H^{1}(\Omega) & \text { in } \Omega, \\
\frac{\partial}{\partial v} \xi_{i}=0 . & \text { on } \Sigma
\end{array}\right\}
$$

reversing sense of time)

Multiply the first equation in (18) by $y_{i}(t ; \mathrm{v})$ and using Green's formula, we obtain the following identity:

Since $\psi_{i} \in H^{1}(\Omega)$, then according to Theorem $1, \mathrm{~b}$ ), system (18) admits an unique strong solution $\xi$. ( after

$$
\begin{aligned}
0 & =\int_{Q}\left[-\frac{\partial \xi_{i}}{\partial t}-\left(A^{*}(t) \xi\right)_{i}\right] y_{i}(t ; \mathrm{v}) d x d t \\
& =-\left.\int_{\Omega} \xi_{i} y_{i}(t ; \mathrm{v})\right|_{0} ^{T} d x+\int_{Q} \xi_{i}\left[\frac{\partial}{\partial t} y_{i}(t ; \mathrm{v})+(A(t) y(t ; \mathrm{v}))_{i}\right] d x d t+\int_{\Sigma} \xi_{i} \frac{\partial y_{i}}{\partial v} d \Sigma \\
& =-<y_{i}(T ; \mathrm{v}), \psi_{i}>+\int_{\Sigma} \frac{\partial \xi}{\partial v} y_{i} d \Sigma
\end{aligned}
$$

implies

$$
\int_{\Sigma} \frac{\partial \xi}{\partial v} y_{i} d \Sigma=0
$$

hence $\frac{\partial \xi}{\partial v}=0$ on $\Sigma$. The Cauchy data of $\xi$ on $\Sigma$ being zero, we conclude ( see [30]) $\xi=0$ and hence $\psi=0$.

\section{Conclusion}

In this study, we have proved the controllability to a special co-operative parabolic systems with Neumann conditions, with deferent cases of observations. Most of the results we described in this paper apply, without any change on the results, to more general parabolic systems involving the following second order operator :

$$
L(x, .)=\sum_{i, j=1}^{n} b_{i j}(x, .) \frac{\partial^{2}}{\partial x_{i} \partial x_{j}}+\sum_{j=1}^{n} b_{j}(x, .) \frac{\partial}{\partial x_{j}}+b_{0}(x, .)
$$

with sufficiently smooth coefficients (in particular, $\left.b_{i j}, b_{j}, b_{0} \in L^{\infty}(Q), b_{j}, b_{0}>0 \quad\right) \quad$ and under the Legendre-Hadamard ellipticity condition

$$
\sum_{i, j=1}^{n} \eta_{i} \eta_{j} \geq \sigma \sum_{i=1}^{n} \eta_{i} \quad \forall(x, t) \in Q,
$$

for all $\eta_{i} \in \Re$ and some constant $\sigma>0$.

In this case, we replace the first eigenvalue of the Laplace operator by the first eigenvalue of the operator $L$ (see [29]).

\section{References}

[1] D.L. Russell, Controllability and stabilizability theory for linear partial differential equations. Recent progress and open questions, SIAM Review 20 (1978), 639-739.
[2] H. T. BANKS, M. Q. JACOBS and R. M. LATINA, The synthesis of optimal controls for linear, time optimal problems with retarded controls, J . Optimization Theory Appl. 8 (1971), $319-360$.

[3] J.L.Lions, "Remarks on approximate controllability, J. Anal. Math, 59, (1992),103-116..

[4] M. C. DELFOUR and S. K. MITTER, Controllability and observability for infinite- dimensional systems, SIAM J. Control Optim. 10 (1972), 329-333.

[5] H. O. FATTORINI, Some remarks on complete montrollability, SIAM J. Control Optim. 4 (1966), 686-694.

[6] H. O. FATTORINI, Boundary control of temperature distributions in a parallelepipedan, SIAM J. Control Optim. 13 (1975), $1-13$.

[7] H. O. FATTORINI and D. L. RUSSELL, Exact controllability theorems for linear parabolitic equations in one space dimension, Arch. Rational Mech. Anal. 43 (1971), 272-292.

[8] J. KLAMKA, Controllability of linear systems with time-variable delays in control, Informat. J . Control 24 (1976), $869-878$.

[9] J. KLAMKA, Absolute controllability of linear systems with time-variable delays in control, Systems Sci. 4 (1978), 43-52.

[10] J. JLLAMKA, Relative controllability of infinite-dimensional systems w ith delays in control, Systems Sci. 4 (1978)) 43-52.

[11] A. W. OLBROT, On the controllability of linear systems with time delays in control, IEEE Trans. Automat. Control 17 (1972), $664-666$.

[12] D. L. RUSSELL, A unified boundary controllability theory for hyperbolic and parabolic partial differential equations, Studies in Appl. Math. 52 (1973), 189-211.

[13] Y. SAKAWA, Controllability for partial differential equations of parabolic type, SIAM J. Control Optim. 12 (1974), 389-400.

[14] T. I. SEIDMAN, Observation and prediction for the heat equation. IV: Patch observability and controllability, SIAM J. Control Optim. 15 (1977), 412-427. 
[15] G. Knowles Time optimal control of parabolic systems with boundary condition involving time delays, Journal of Optimiz.Theor. Applics, 25,( 1978), 563-574 .

[16] R. TRIGGIANI, Controllability and observability in Banach space with bounded operators, SIAM J. Control Optim. 13 (1975), 462-491.

[17] R. TRIGGIANI, Extensions of rank conditions for controllability and observability to Banach space and unbounded operators, SIAM J. Control Optim. 14 (1976), $313-338$.

[18] R. TRIGGIANI, On the lack of exact controllability for mild solutions in Banach space, J. Math. Anal. Appl. 50 (1975) $438-446$.

[19] J.-L. Lions, Exact controllability, stabilizability and perturbations for distributed systems, SIAM Review, 30 (1988) $1-68$.

[20] J.L.Lions, Optimal control of systems governed by partial differential equations, Springer-verlag, Band 170, (1971).

[21] J.L.Lions and E.Magenes, " Non homogeneous boundary value problem and applications, I, II, Spring-Verlage, New York, (1972).

[22] H. O. Fattorini, Infinite dimensional Optimization Theory and Opotimal Control, Cambridge Univ. Prees (1998).

[23] H.A. El-Saify, H.M. Serag and M.A.Shehata, Time-optimal control for co-operative hyperbolic systems Involving Laplace operator. Journal of Dynamical and Control systems. 15,3,(2009),405-423.
[24] M.A.Shehata, Some time-optimal control problems for $n \times n$ co-operative hyperbolic systems with distributed or boundary controls. Journal of Mathematical Sciences: Advances and Applications. vol 18, No 1-2,(2012),63-83.

[25] M.A.Shehata, Time -optimal control problem for $n \times n$ co-operative parabolic systems with control in initial conditions, Advances in Pure Mathematics Journal , 3, No 9A,(2013),38-43.

[26] M.A.Shehata, Dirichlet Time-Optimal Control of Co-operative Hyperbolic Systems Advanced Modeling and Optimization Journal. Volume 16, Number 2, (2014),355-369.

[27] Byung Soo Lee, Mohammed Shehata, Salahuddin , Time -optimal control problem for $n \times n$ co-operative parabolic systems with strong constraint control in initial conditions, Journal of Science and Technology, Vol.4 No.11,(2014)..

[28] R.A. Adams, Sobolev Spaces, Academic Prees, New York, (1975).

[29] J. Fleckinger, J. Hern $a^{\prime}$ ndez and F.DE. Th $e^{\prime}$ lin, On the existence of multiple principal eigenvalues for some indefinite linear eigenvalue problems, Rev.R.Acad.Cien.Serie A.Mat. 97,2 (2003), 461-466.

[30] H. Tanabe, On differentiability and analyticity of eighted elliptic boundary value problems, Osaka Math.Journal ,2 (1965),163-190. 